\title{
Mathematical model of centrifugal compressor vaneless diffuser based on CFD calculations
}

\author{
Olga Solovyeva ${ }^{1, *}$, and Aleksandr Drozdov ${ }^{1}$ \\ ${ }^{1}$ Peter the Great St.Petersburg Polytechnic University St.Petersburg, Polytechnicheskaya, 29, Russia
}

\begin{abstract}
The approximate engineering techniques based on mathematical modelling are used in centrifugal compressor design. One of such methods is the well-proven Universal Modelling Method, developed in the scientific and research laboratory "Gas dynamics of turbo machines", SPbPU. In the modern version of the compressor model, vaneless diffusers mathematical model was applied based on a generalization of the CFD calculations. The mathematical model can be used for vaneless diffusers with a relative width in the range of $1.4-10.0 \%$, with a radial length up to 2.0 , in the range of inlet flow angles 10 to 90 degrees, the inlet velocity coefficient in the range of $0.39-0.82$, Reynolds number varying from 87500 to 1030000 . The model was also used for calculating low-flow-rate model stages with narrow diffusers with diffusers' relative width in the range of $0.5-2.0 \%$. The mathematical model showed lesser accuracy. To widen the model applicability, new series of CFD-calculations were executed. A series of vaneless diffusers was designed with relative width in the range of $0.6-1.2 \%$, The gas-dynamic characteristics of loss coefficients and outlet flow angle versus inlet flow angle of diffuser were calculated. Regression analysis was used to process the calculated data. System of algebraic equations linking geometric, gas-dynamic parameters and similarity criteria was developed. The obtained equations are included in a new mathematical model of the Universal Modelling Method.
\end{abstract}

\section{Introduction}

CFD calculations are widely used for the calculation and design of centrifugal compressors and their elements [14], but the problem of verification of the calculated data is important. The calculation results largely depend on the object of research, applied software packages, selected boundary conditions, etc. Some foreign authors claim good agreement between the calculated and experimental data [5-8]. In many cases, the authors either do not indicate the calculation methodology, or use programs of their own design. Other authors point to a number of unsolved problems with the verification of CFD calculations. The main problems are associated with the calculation of the centrifugal compressor impellers. Calculations for various CFD programs overestimate the loading factor by $7-12 \%$, depending on the type of impeller, and shift the calculated gasdynamic characteristics of efficiency to high flow rates.

The authors' experience confirms the presence of such problems [10-13]. In our opinion, solving the problem of overstating the loading factor will also lead to the matching of the ranges of the calculated and measured efficiency characteristics. The experience of the R\&D Laboratory "Gas Dynamics of Turbomachines" in the application of CFD calculations to the modeling of stator elements of centrifugal stages shows good results [14-15]. CFD calculations have been applied in the implementation of gas-dynamic projects of centrifugal compressors and replaceable flow paths in the interests of industrial partners. In particular, the shape of the VLD and the crossover was optimized. Based on this experience, O.A. Solovyeva as part of a $\mathrm{PhD}$ thesis research [16] developed a new mathematical model of VLD. Its features are presented below.

\section{Materials and methods}

\subsection{The mathematical model of VLD, based on a generalization of the computational experiment for VLD with $b_{3} / D_{2}=\mathbf{0 . 0 1 4}-\mathbf{0 . 1 0 0}$ (model No. 1)}

At the first stage, researches were conducted on the method of setting, conducting and processing the results of CFD calculations of VLD [15]. Recommendations are made on choosing the density of the computational grid and the turbulence model. Before conducting virtual aerodynamic tests of the VLD series, it is necessary to make sure that the flow pattern calculated using the CFD program corresponds to the physical meaning, and the integral characteristics match the experimental results with the required accuracy. For this, a comparison of the results of a computational experiment with the known experimental data was performed. Figure 1 shows a comparison of VLD characteristics calculated by the ANSYS CFX program and experimental ones tested together with impellers [16]. For comparison, the

Corresponding author: Solovyeva.oa@yandex.ru 
calculated diffusers closest in parameters were chosen $b_{3} / D_{2}=0.014,0.016,0.033,0.038$, with radial length $D_{4} / D_{2}=1.6$, Mach number $M_{c 2}=0.75$ (where $b_{3}$ is VLD width, $D_{2}$ is impeller diameter, $D_{4}$ is VLD outlet diameter, $M_{c 2}$ is Mach number). In experimental and computational researches, the pressure coefficient increases with increasing ratio $c_{r 2} / c_{u 2}$ (where $c_{r 2}$ is the radial component of absolute velocity, $c_{u 2}$ is the circumferential component of velocity, $\alpha_{2}$ is the flow angle) $\left(c_{r 2} / c_{u 2}=\operatorname{tg} \alpha_{2}\right)$, i.e., the diffuser works less and less efficiently as the flow direction approaches the tangential one. Some of the results, which correspond to a small value $c_{r 2} / c_{u 2}$, were obtained with a rotating stall; accordingly, these results are less reliable [16]. As a result, for a small value $c_{r 2} / c_{u 2}$, the difference between the experimental $\left(b_{3} / D_{2}=0.0390\right)$ and calculated $\left(b_{3} / D_{2}=0.0380\right)$ characteristics is large. Characteristics calculated by the ANSYS CFX program are higher than the experimental characteristics. The difference is explained by the fact that the experimental data presented in [16] relate to stages where mixing losses occur in the VLD, caused by the appearance of a "wake" in the impeller. The uneven flow pattern at the impeller outlet also increases VLD losses. In the calculation of an isolated VLD, there is no negative influence of the impeller.

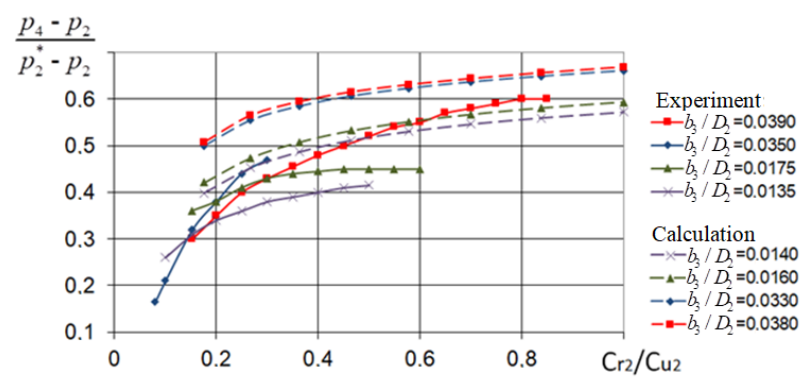

Fig. 1. Comparison of experimental and calculated pressure recovery coefficients in a vaneless diffuser depending on the parameter $c_{r 2} / c_{u 2}$ for different values of the relative width of the diffuser. Experiment (solid line): $b_{3} / D_{2}=0.0135,0.0175$,

0.0350, 0.0390; calculation (dashed line): $b_{3} / D_{2}=0.0140$, $0.0160,0.0330,0.0380$.

Comparison with the provisions of the theory showed the regularity of the flow of gas-dynamic characteristics, and with well-known experiments - a good agreement on the flow structure. This allowed us to draw conclusions about the reliability of the data obtained as a result of the calculation, and the validity of replacing the physical experiment in the research of VLD by a computational experiment. The flow and gas-dynamic characteristics of the VLD with a relative width $b_{3} / D_{2}=$ $0.014-0.100$, with a radial length $\mathrm{D}_{4} / \mathrm{D}_{2}$ up to 2.0 , and in the range of inlet flow angles $\alpha_{2}=10-90^{\circ}[15,16]$. Similarity criteria varied between $\lambda_{c 2}=0.39-0.82$,
$\mathrm{Re}_{b 2}=87500-1030000$ (where $\lambda_{c 2}$ is velocity coefficient, $\operatorname{Re}_{b 2}$ is Reynolds number). The approximation of the computational experiment was performed using the EXCEL program by the method of regression analysis. To calculate the flow parameters at the VLD outlet, we need to know any two of five coefficients - efficiency, loss coefficient, velocity ratio, recovery coefficient, and outlet flow angle. The loss factor and the outlet flow angle from the VLD were selected as modeling objects:

$$
\zeta, \alpha_{4}=f\left(\bar{b}_{3}, \bar{D}_{4}, \alpha_{2}, \lambda_{c 2}, \operatorname{Re}_{b 2}, \bar{\kappa}_{\text {roug }}\right)
$$

where $k_{\text {rough }}$ is relative surface roughness, $\zeta$ is the loss coefficient.

The loss coefficient was simulated in three stages: first, the dependence $\zeta=f\left(\bar{b}_{3}, \alpha_{2}, \lambda_{c 2}\right)$ was approximated, then correction factors were introduced that take into account the influence of the VLD length, Reynolds criterion, and relative roughness. The correction for the influence of the Reynolds criterion takes into account the surface roughness if the surface is not hydraulically smooth. The general structure of the formula is:

$$
\zeta=\frac{A \cdot \alpha^{B} \cdot K_{D 4}}{K_{\mathrm{Re}, k_{\text {roug }}}}
$$

where: $A=c \cdot\left(b_{3} / D_{2}\right)^{2}+d \cdot\left(b_{2} / D_{2}\right)+e$;

$$
\begin{aligned}
& c=2312.7 \cdot \lambda_{c 2}^{2}-2234.9 \cdot \lambda_{c 2}+830.63 \\
& d=-207.36 \cdot \lambda_{c 2}^{2}+266.98 \cdot \lambda_{c 2}-88.333 \\
& e=6.4922 \cdot \lambda_{c 2}^{2}-6.9342 \cdot \lambda_{c 2}+3.5363 \\
& B=f \cdot\left(b_{3} / D_{2}\right)^{2}+g \cdot\left(b_{3} / D_{2}\right)+h ; \\
& f=-124.32 \cdot \lambda_{c 2}^{2}+186.87 \cdot \lambda_{c 2}-17.242 \\
& g=19.241 \cdot \lambda_{c 2}^{2}-29.915 \cdot \lambda_{c 2}-3.3289 ; \\
& h=-0.6906 \cdot \lambda_{c 2}^{2}+0.6577 \cdot \lambda_{c 2}-0.527
\end{aligned}
$$

To account for the VLD length, a rather complicated system of approximating formulas was also obtained:

$$
K_{D 4}=j \cdot\left(D_{4} / D_{2}\right)^{2}+k \cdot\left(D_{4} / D_{2}\right)+l,
$$

$$
\begin{aligned}
& \text { where: } j=m \cdot\left(b_{3} / D_{2}\right)^{2}+n \cdot\left(b_{3} / D_{2}\right)+p ; \\
& m=3455.2 \cdot \lambda_{c 2}^{2}-3513.1 \cdot \lambda_{c 2}+669.16 ; \\
& n=-443.08 \cdot \lambda_{c 2}^{2}+427.91 \cdot \lambda_{c 2}-74.406 ; \\
& p=8.756 \cdot \lambda_{c 2}^{2}-8.6285 \cdot \lambda_{c 2}+0.5291 \\
& k=r \cdot\left(b_{3} / D_{2}\right)^{2}+s \cdot\left(b_{3} / D_{2}\right)+t \\
& r=-12860 \cdot \lambda_{c 2}^{2}+13344 \cdot \lambda_{c 2}-2726.1 ; \\
& s=1633.8 \cdot \lambda_{c 2}^{2}-1613.6 \cdot \lambda_{c 2}+306.13 ; \\
& t=-31.076 \cdot \lambda_{c 2}^{2}+31.02 \cdot \lambda_{c 2}-1.7055 \\
& l=u \cdot\left(b_{3} / D_{2}\right)^{2}+v \cdot\left(b_{3} / D_{2}\right)+w ; \\
& u=11401 \cdot \lambda_{c 2}^{2}-11984 \cdot \lambda_{c 2}+2574.4 \\
& v=-1439.3 \cdot \lambda_{c 2}^{2}+1440.6 \cdot \lambda_{c 2}-290.69 ; \\
& w=26.521 \cdot \lambda_{c 2}^{2}-26.682 \cdot \lambda_{c 2}+2.2384
\end{aligned}
$$




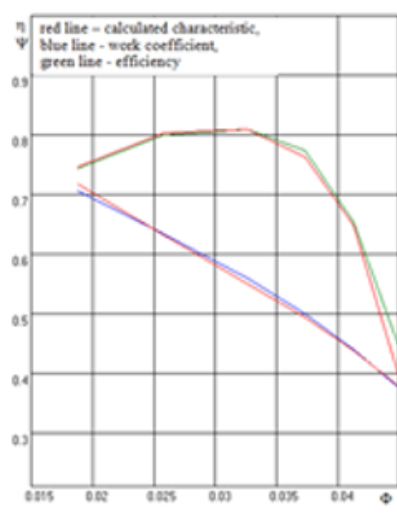

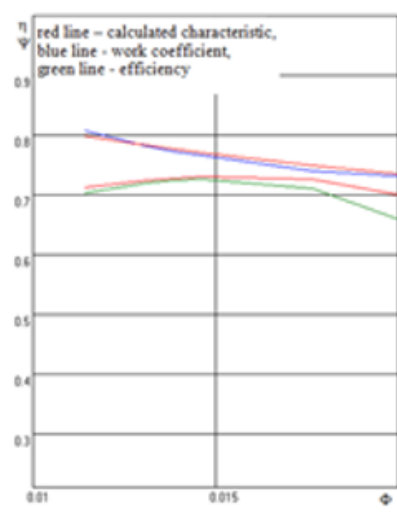

b

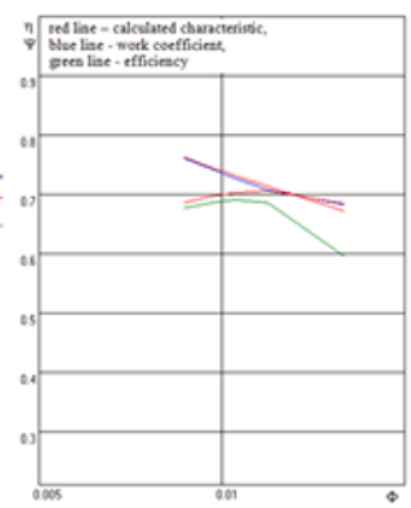

Fig. 2. Comparison of calculated and experimental data for the stage a) $0028-056-0373 \Phi_{\text {des }}=0.028, \psi_{T \text { des }}=0.56, \bar{D}_{\text {hub }}=$ $\left.0.373, \bar{b}_{3}=0.033 ; \bar{D}_{4}=1.45, M_{u}=0.60, \operatorname{Re}_{u}=4.8 \cdot 10^{6} ; \mathrm{b}\right) \mathrm{XXX} 3-\mathrm{Q}$ with parameters: $\Phi_{\text {des }}=0.0203, \psi_{T \text { des }}=0.69, \bar{D}_{h u b}=$

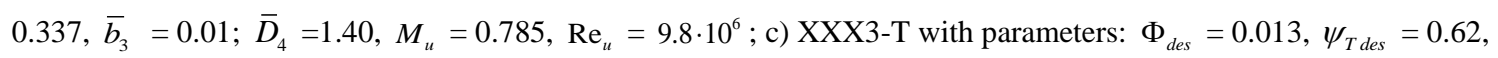
$\bar{D}_{h u b}=0.337, \bar{b}_{3}=0.008 ; \bar{D}_{4}=1.40, M_{u}=0.803, \operatorname{Re}_{u}=10 \cdot 10^{6}$.

Correction for the influence of the Reynolds criterion - relative roughness - was made by analogy with the resistance coefficient for the plate, but taking into account the specifics of the formulation of the computational experiment. For a hydraulically smooth surface:

$$
K_{\mathrm{Re}}=\frac{\lambda_{\text {calc }}}{\lambda_{M M}}=\frac{0.0032+\frac{0.221}{\left(8.73 \cdot 10^{6} \cdot \bar{b}_{3}\right)^{0.237}}}{0.0032+\frac{0.221}{\mathrm{Re}_{b}^{0.237}}} ;
$$

- for rough surface:

$$
K_{u u}=\frac{\lambda_{\text {calc }}}{\lambda_{M M}}=\left(0.0032+\frac{0.221}{\left(8.73 \cdot 10^{6} \cdot \bar{b}_{3}\right)^{0.237}}\right)\left(2 \lg \frac{2}{\bar{k}_{\text {roug }}}+1.74\right)^{2} \text {. }
$$

In the calculations, we choose the formula, in which the loss coefficient is greater. The system of equations is characterized by a good accuracy of $98.6 \%$. The approximation for calculating the outlet flow angle from the VLD is performed using the same method as for the loss coefficient - the method of regression analysis. The calculated data are approximated by a power polynomial of the following form:

$$
\alpha_{4}=\alpha_{2}+a \cdot \alpha_{2}^{2}+b \cdot \alpha_{2}+c,
$$

where: $a=d \cdot \lambda_{c 2}+e$;

$$
\begin{aligned}
& d=0.0013 \ln \left(b_{3} / D_{2}\right)+0.01 \\
& e=-0.0021 ; \\
& b=f \cdot \lambda_{c 2}+g ; f=-0.13 \ln \left(b_{3} / D_{2}\right)-0.9844 ; \\
& g=0.0563 \ln \left(b_{3} / D_{2}\right)+0.3345 \\
& c=h \cdot \lambda_{c 2}+i ; h=2.208 \ln \left(b_{3} / D_{2}\right)+9.7999 ; \\
& i=-5.137 \ln \left(b_{3} / D_{2}\right)-13.136
\end{aligned}
$$

In the mathematical model there are no empirical coefficients that need to be identified. In addition, due to the fact that the entire diffuser is calculated, and not 20 of its parts, as in previous versions of the model, the calculation speed increases. The proposed formulas are implemented using the gas-dynamic function of the current density [16]. The result is an equation containing one unknown - the velocity coefficient $\lambda_{c 4}$ at the VLD outlet:

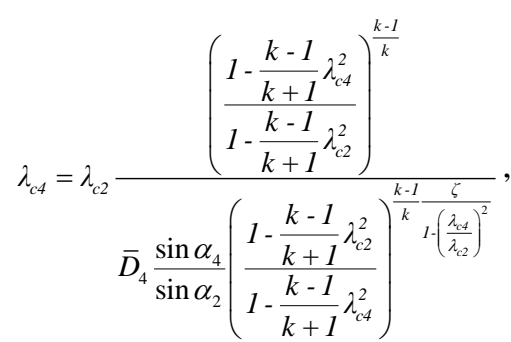

where $k$ is isentropic coefficient. The equation is solved by bruteforcing. Further calculation is based on the wellknown equations of thermogasdynamics. The developed mathematical model was applied in the 8th version of the Universal Modeling Method programs. A comparison of the calculated by the 8th version of the mathematical model and the measured gas-dynamic characteristics was carried out for model stages of the 20CE series with VLD. The range of basic design parameters for model stages: $\Phi_{\text {des }}=0.028-0.080, \psi_{\text {Tdes }}=0.40-0.67$, $\bar{D}_{\text {hub }}=0.25-0.373, b_{2} / D_{2}=0.03-0.061 ; \bar{D}_{4}=1.42-$ $1.60, M_{u}=0.60-0.80, \operatorname{Re}_{u}=5.9 \cdot 10^{6}-8.0 \cdot 10^{6}$ (where $\bar{D}_{h u b}$ is relative hub diameter, $\Phi_{\text {des }}$ is design relative flow rate coefficient, $\psi_{T \text { des }}$ is design loading factor). Comparison showed the average error in modeling the design (maximum) efficiency of $0.977 \%$, the average error at five points (except for the point of highest flow rate) being $1.27 \%$. An example of a comparison is shown in the figure $2 \mathrm{a}$.

\subsection{Application of the model for Clarke model stages}

The second object of assessing the applicability and effectiveness of the developed VLD model was the 

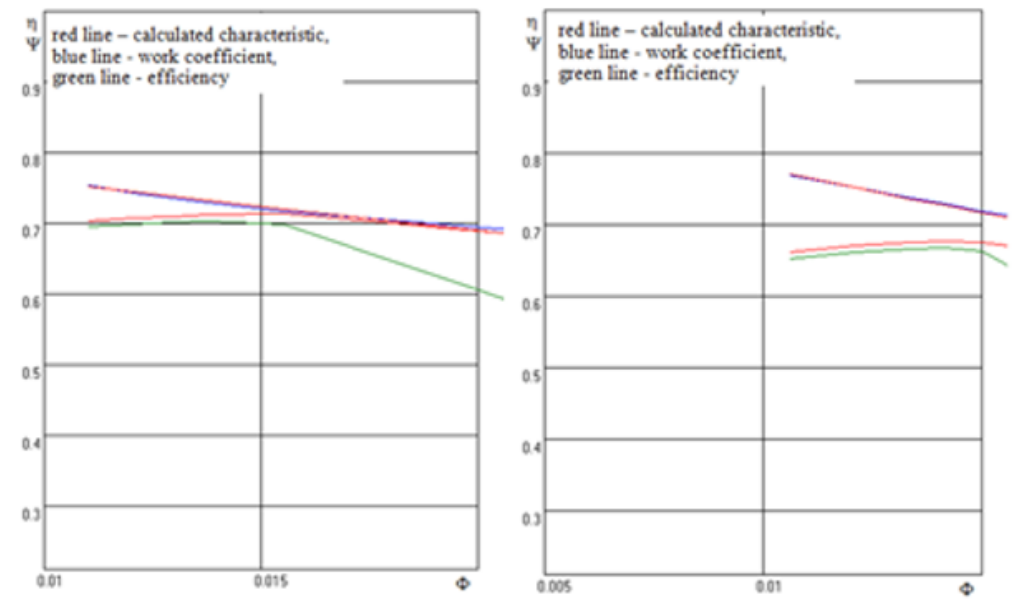

Fig. 3. Comparison of calculated and experimental data for stage XXX3-S with parameters: $\Phi_{\text {des }}=0.015, \psi_{\text {Tdes }}=0.63$,

$\bar{D}_{h u b}=0.337, \bar{b}_{3}=0.008 ; \bar{D}_{4}=1.40, M_{u}=0.803, \operatorname{Re}_{u}=10 \cdot 10^{6}$ (left) and stage XXX3-U with parameters: $\Phi_{\text {des }}=0.011$,

$\psi_{T \text { des }}=0.62, \bar{D}_{h u b}=0.337, \bar{b}_{3}=0.0057 ; \bar{D}_{4}=1.40, M_{u}=0.803, \operatorname{Re}_{u}=10 \cdot 10^{6}$.

Clarke series of low-consumption model stages, designed in the 1950s. The range of basic design parameters for model stages of the Clarke company is: $\Phi_{\text {des }}=0.007-0.024, \psi_{\text {Tdes }}=0.60-0.69, \bar{D}_{\text {hub }}=0.3214$ $-0.3375, b_{2} / D_{2}=0.0094-0.0476 ; \bar{D}_{4}=1.33-1.4$, $M_{u}=0.366-0.843, \quad \operatorname{Re}_{u}=4.8 \cdot 10^{6}-1.1 \cdot 10^{7}$. Comparison of calculated and experimental data showed an average error of modeling the design (maximum) efficiency of $1.15 \%$, an average error of five points (except for the point of highest flow rate) being $2.15 \%$. An example of comparison of calculated and measured gas-dynamic characteristics is presented in the figures $2 \mathrm{~b}), \mathrm{c}$ ) and 3 . We can see that for low-flow-rate stages, there is a tendency to overestimate the calculated efficiency compared to the measured one. One reason for this is the underestimation of the VLD loss coefficient. The stages considered have VLD with a width of $\bar{b}_{3}=$ $0.005-0.019$, which for most stages extends beyond the width of the VLD involved in virtual wind tunnel tests and the creation of a VLD mathematical model.

This shows that it is incorrect to extrapolate previously calculated data to narrower VLD. In this

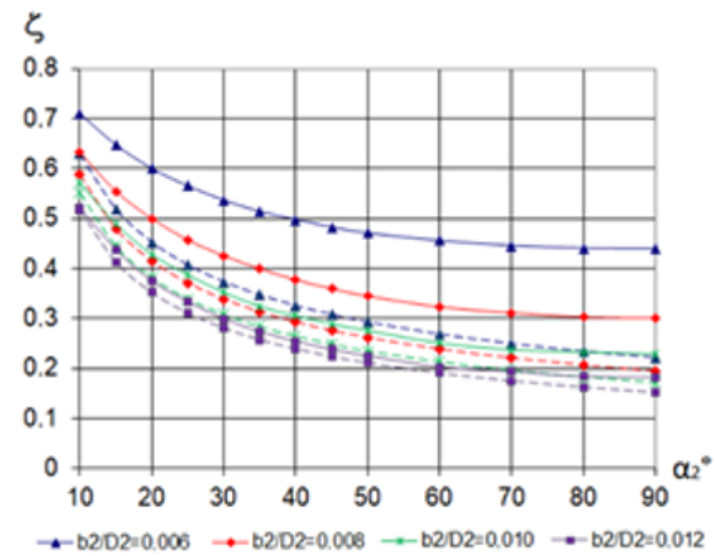

regard, it became necessary to refine the already existing mathematical model by including narrow VLD in the sample for approximation. This assumption required verification. The reason for the difference in the calculated and experimental characteristics may also be underestimation of the impact of losses in other elements of the flow part. Thus, in low-flow stages, friction losses (due to the fact that the channels are narrow and long) have a greater impact on the reduction of efficiency than mixing losses. Since there are no element-by-element characteristics for Clarke model stages, it is possible to verify the conclusions made only with the help of CFD calculations. For this purpose, four diffusers were calculated with a relative width corresponding to that used in the Clarke stages $b_{3} / D_{2}=0.006,0.008,0.010$, 0.012 .

Calculation setup corresponds to the above recommendations. For these objects, the characteristics of the loss coefficient and the change of the flow angle in the VLD were calculated. They were compared with the calculation using formulas (2) and (7), see figure 4.

In the calculated range of narrow diffusers, the width decreases by 2 times, while the loss coefficient increases

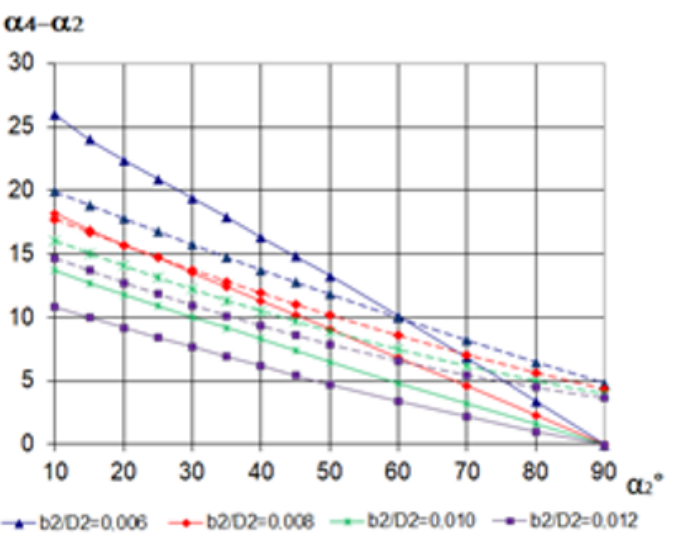

Fig. 4. Comparison of approximating (dashed line) and calculated (solid line) values of VLD loss coefficient (left) and $\alpha_{4}-\alpha_{2}$ (right) with $b_{3} / D_{2}=0.006,0.008,0.010,0.012, \bar{D}_{4}=1.60, \lambda_{c 2}=0.64$ 


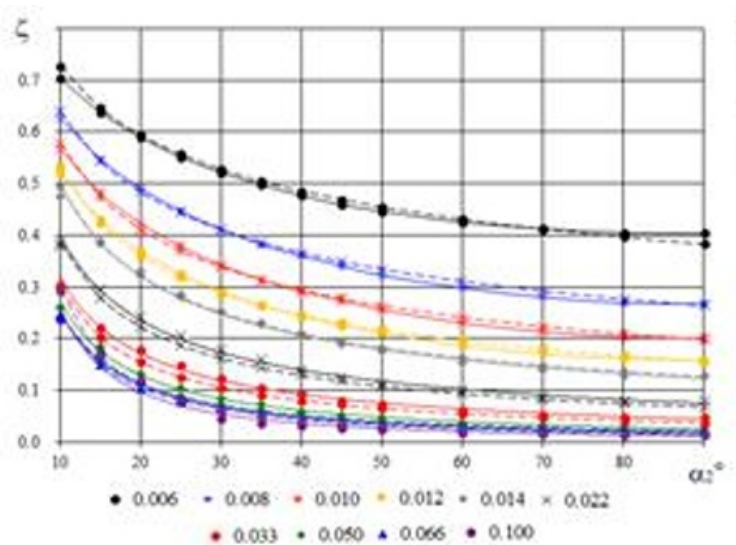

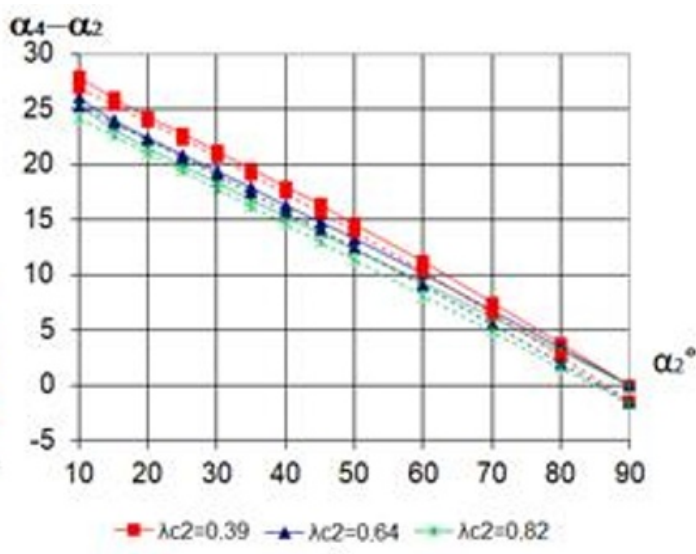

$\mathrm{b}$

Fig. 5. Comparison of calculated and approximating values of VLD a) loss coefficients depending on the angle $\alpha_{2}$.

$D_{4} / D_{2}=1.6, \lambda_{c 2}=0.39$; b) $\alpha_{4}-\alpha_{2}$ depending on the inlet flow angle at $\lambda_{c 2}=0.39,0.64,0.82 . b_{3} / D_{2}=0.006 ; D_{4} / D_{2}=1.6$.

Solid lines - calculation, dashed lines - approximation.

by 1.5 times, the efficiency decreases by 1.6 times. For comparison, in the previously calculated series of diffusers, the width decreased by 7.15 times, and the loss factor increased by 2.6 times. This shows that for narrow diffusers, width has a greater influence on the value of the loss coefficient. If we apply the previously proposed mathematical model for narrow vaneless diffusers, then for a diffuser with a relative width $b_{3} / D_{2}=0.006$, the model understates the loss coefficient by $1.13-2.0$ times, and the outlet flow angle by $1.24-0.95$ times for the inlet flow angles of 10 and 90 degrees, respectively.

\subsection{Objects}

To increase the accuracy of modeling low-flow-rate stages, additional calculations of narrow VLD were performed in the ANSYS CFX program. The characteristics of diffusers with a relative width $b_{3} / D_{2}=0.006,0.008,0.010,0.012$ and with a radial length $D_{4} / D_{2}=1.6-2.0$ in the range of velocity coefficients $\lambda_{c 2}=0.39-0.82$ and the corresponding Reynolds numbers $\operatorname{Re}_{b 2}=36800-73$ 600. Calculations were made at inlet flow angles $\alpha_{2}=10^{\circ}-90^{\circ}$.

\subsection{The mathematical model of VLD, based on a generalization of the computational experiment for VLD with $b_{3} / D_{2}=\mathbf{0 . 0 0 6 - 0 . 1 0 0}$ (model No. 2)}

In general, the approximating dependence for the loss coefficient $\zeta=f\left(\bar{b}_{2}, \bar{D}_{4}, \alpha_{2}, \lambda_{c 2}, \operatorname{Re}, \bar{k}_{\text {roug }}\right)$ is presented in the same form as for model No. 1 , however, the system of approximating formulas has a completely different structure and coefficients. At the first step, the influence of $b_{3} / D_{2}, \alpha_{2}, \lambda_{c 2}$ was approximated at a fixed value of the relative length of the diffuser $D_{4} / D_{2}=1.6$ :

$$
\zeta=\frac{A \cdot \alpha^{B} \cdot K_{D 4}}{K_{\mathrm{Re}, k_{\text {roug }}}}
$$

where:

$$
\begin{aligned}
& A=c \cdot\left(b_{3} / D_{2}\right)^{4}+d \cdot\left(b_{3} / D_{2}\right)^{3}+e \cdot\left(b_{3} / D_{2}\right)^{2}+h \cdot\left(b_{3} / D_{2}\right)+i ; \\
& c=111633 \cdot \lambda_{c 2}^{2}-208134 \cdot \lambda_{c 2}-184432 \\
& d=64704 \cdot \lambda_{c 2}^{2}-61330 \cdot \lambda_{c 2}+71806 ; \\
& e=-10988 \cdot \lambda_{c 2}^{2}+12468 \cdot \lambda_{c 2}-7411.4 ; \\
& h=465.59 \cdot \lambda_{c 2}^{2}-499.03 \cdot \lambda_{c 2}+245.24 ; \\
& i=-1.7437 \cdot \lambda_{c 2}^{2}+2.468 \cdot \lambda_{c 2}-0.17 ; B=f \cdot \ln \left(b_{3} / D_{2}\right)+g ; \\
& f=0.0796 \cdot \lambda_{c 2}^{2}-0.194 \cdot \lambda_{c 2}-0.2775 ; \\
& g=-0.7042 \cdot \lambda_{c 2}-1.6742 .
\end{aligned}
$$

Corrections for the influence of the Reynolds criterion - relative roughness and relative length of the diffuser are made by analogy with model No. 1, using the formulas (3), (4), (5).

The approximation for calculating the outlet flow angle from the VLD is performed differently for the VLD range with a relative width $0.006 \leq b_{3} / D_{2} \leq 0.012$ and $0.012<b_{3} / D_{2} \leq 0.100$.

For VLD with relative width $0.006 \leq b_{3} / D_{2} \leq 0.012$ :

$$
\alpha_{4}=\alpha_{2}+A \cdot \alpha_{2}^{2}+B \cdot \alpha_{2}+C
$$

where: $A=d \cdot \lambda_{c 2}+e$;

$$
\begin{aligned}
& d=0.0036 \cdot \ln \left(b_{3} / D_{2}\right)+0.0199 ; \\
& e=-0.15 \cdot\left(b_{3} / D_{2}\right)-0.0004 ; B=f \cdot \lambda_{c 2}+g ; \\
& f=-0.431 \cdot \ln \left(b_{3} / D_{2}\right)-2.2826 ; \\
& g=0.4487 \cdot \ln \left(b_{3} / D_{2}\right)+2.0442 ; C=h \cdot \lambda_{c 2}+i ; \\
& h=6.8893 \cdot \ln \left(b_{3} / D_{2}\right)+29.521 ; \\
& i=-27.87 \cdot \ln \left(b_{3} / D_{2}\right)-110.55 .
\end{aligned}
$$

For diffusers with a relative width $0.012<b_{3} / D_{2} \leq 0.100$, the model for calculating the outlet flow angle remains the same, the formula (6).

The resulting system of 42 algebraic equations is characterized by a good accuracy of $98.9 \%$, figure 5 . 


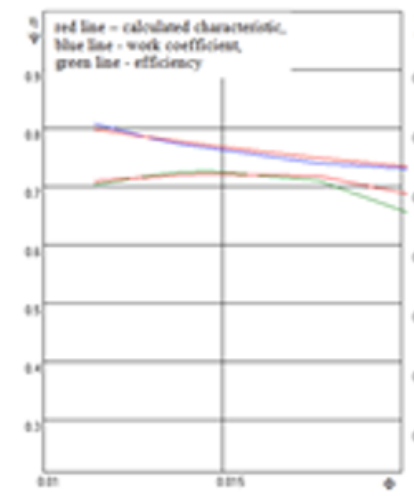

a

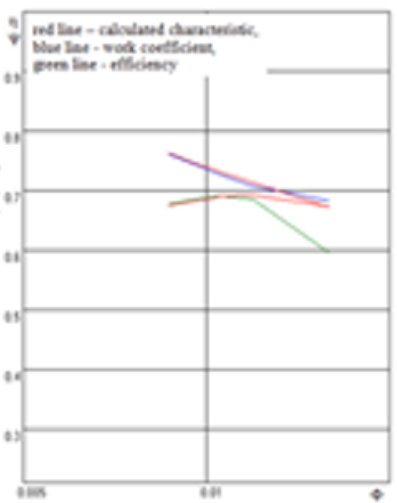

b

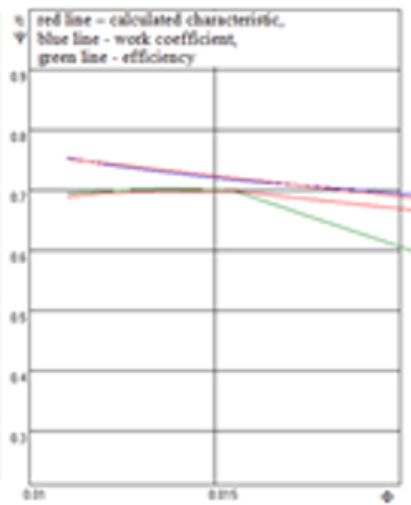

c

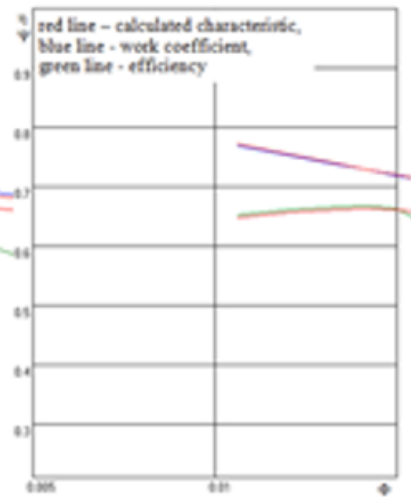

d

Fig. 6. Comparison of calculated and experimental data for stage a) XXX3-Q with parameters: $\Phi_{\text {des }}=0.0203, \psi_{\text {Tes }}=0.69$, $\left.\bar{D}_{h u b}=0.337, \bar{b}_{3}=0.01 ; \bar{D}_{4}=1.40, M_{u}=0.785, \operatorname{Re}_{u}=9.8 \cdot 10^{6} ; \mathrm{b}\right)$ stage XXX3-T with parameters: $\Phi_{\text {des }}=0.013, \psi_{T \text { des }}=$ $\left.0.62, \bar{D}_{h u b}=0.337, \bar{b}_{3}=0.008 ; \bar{D}_{4}=1.40, M_{u}=0.803, \operatorname{Re}_{u}=10 \cdot 10^{6} ; \mathrm{c}\right) \mathrm{XXX} 3-\mathrm{S}$ with parameters: $\Phi_{\text {des }}=0.015$, $\psi_{T \text { des }}=0.63, \bar{D}_{h u b}=0.337, \bar{b}_{3}=0.008 ; \bar{D}_{4}=1.40, M_{u}=0.803, \operatorname{Re}_{u}=10 \cdot 10^{6}$; stage XXX3-U with parameters: $\Phi_{d e s}=$ $0.011, \psi_{T d e s}=0.62, \bar{D}_{h u b}=0.337, \bar{b}_{3}=0.0057 ; \bar{D}_{4}=1.40, M_{u}=0.803, \operatorname{Re}_{u}=10 \cdot 10^{6}$.

\section{Results and discussion}

\subsection{Application of model No. 2 in the programs of the Universal Modelling Method}

Formulas (8)-(9) were introduced into the mathematical model of the Universal Modeling Method. Since the proposed VLD model both by formulas (3) (6) and by formulas (8) - (9) does not imply the presence of empirical coefficients, identification was not required. A comparison was made between the calculated and experimental gas-dynamic characteristics for model stages 20CE and model stages of the Clarke series. For model stages 20CE, the comparison showed an average error of modeling the design (maximum) efficiency of $1.01 \%$, an average error of five points (except for the point of highest flow rate) of $1.31 \%$.

For Clarke model stages, a comparison of the calculated and experimental data showed an average error of modeling the design (maximum) efficiency of $0.99 \%$, an average error of five points (except for the point of highest flow rate) of $1.97 \%$. Figure 6 shows a comparison of the calculated and measured gas-dynamic characteristics of the same Clarke model stages, which were presented above in figures 2 - 3. They clearly show the improvement of the modeling accuracy of the gasdynamic characteristics of low-flow-rate model stages.

The results showed a slight increase in the error in calculating the characteristics of model stages 20CE (about $0.05 \%$ ) and a decrease in the error in calculating Clarke model stages (about $0.2 \%$ ). This confirms the accuracy of the analysis and the effectiveness of the developed approximating dependencies.

\section{Conclusion}

The mathematical model of flow parameters calculation in VLD with relative width $b_{3} / D_{2}=0.014-0.100$ created in [16] was applied for calculation of mediumflow model stages of 20CE series (relative width of VLD $\left.b_{3} / D_{2}=0.030-0.061\right)$ and low-flow model stages by Clarke Tooling (relative width of VLD $b_{3} / D_{2}=0.005-$ 0.019). Application experience has shown good match between calculated and experimental gas dynamic characteristics of medium-flow model stages and overestimation of efficiency for low-flow stages. Comparison of the results of CFD calculations of narrow VLD with the calculation using approximating formulas confirmed the assumption that the reason for the overestimation of the efficiency of low-flow stages is the insufficient accuracy of modeling the loss coefficient and the outlet angle of the flow in narrow VLD. A series of CFD calculations of narrow VLD in the range $b_{3} / D_{2}=0.006-0.012$ was performed and the obtained characteristics of the loss coefficient and the change of the flow angle were approximated. The mathematical model of VLD created on the basis of new approximating formulas allowed to increase accuracy of efficiency modeling of low-flow stages by $16 \%$ for the mode with the maximum efficiency and by $9 \%$ for the characteristic as a whole, without losing in accuracy of modeling of medium-flow stages at the same time.

\section{Acknowledgemetns}

The research was performed by a Grant of the President of the Russian Federation for young PhD MK1893.2020.8.

The calculations were carried out using the supercomputer center "Polytechnic" SPbPU. 


\section{References}

[1] A. Startsev, Yu. Fokin, Eu. Steshakov, CFD design and analysis of a compact single-spool compressor for a heavy transport helicopter's powerplant, 29th congress of the international council of the aeronautical sciences (St. Petersburg, Russia, 2014)

[2] C. Xu, W.J. Chen, Computational analysis on a compressor blade, Int. Conf. On Jets, Wakes and Separated Flow, Proceedings of Int. Conf. On Jets, Wakes and Separated Flow (Toba-shi, Mie, Japan, 2005)

[3] Jindra Kosprdova, Jiři Oldřich, The Development of Centrifugal Turbo Compressor Stage Using CFD, The 20th International Conference on Hydraulics and Pneumatics (Prague, 2008)

[4] R. Puzyrewski, Y. Galerkin, P. Flaszynski, Direct and inverse numerical calculation for the tested centrifugal impeller, XI Internationale tagung forschung praxis und didaktik im modernen maschinenbau (Germany, Stralsund 2001)

[5] M. Mosdzien, M. Enneking, A. Hehn, D. Grates, P. Jeschke, Journal of the Global Power and Propulsion Society 2, 1 (2018)

[6] J.M. Sorokes, B.R. Hutchinson, The Practical Application of CFD in the Design of Industrial Centrifugal Compressors, ASME International Mechanical Engineering Congress and Exposition, Challenges and Goals in Pipeline Compressors, edited by A. Engeda (USA, Florida, 2000)

[7] J.M. Sorokes, Sidestream optimization through the use of computational fluid dynamics and model testing turbomachinery, sympos. Proceed. Texas: A\&M (2000)

[8] G.E. Guidotti, Towards Centrifugal Compressor Stages Virtual Testing: Ph.D. thesis (Università degli Studi di Bologna, 2014)

[9] R. Matas, T. Syka, L. Hurda, EPJ Web of Conferences 180, 02060 (2018)

[10] Y. Galerkin, I. Voinov, A. Drozdov, Comparison of CFD-calculations of centrifugal compressor stages by NUMECA Fine/Turbo and ANSYS CFX programs, International Conference on Compressors and their Systems 2019, IOP Conf. Series: Materials Science and Engineering (London, 2017)

[11] A.I. Borovkov, I.B. Voinov, M.A. Nikitin, Yu.B. Galerkin, A.F. Rekstin, A.A. Drozdov, Experience of performance modeling the singlestage pipeline centrifugal compressor, Oil and Gas Engineering (OGE-2019), AIP Conf. Proc. 2007 (Omsk, 2019)

[12] A.I. Borovkov, I.B. Voinov, Yu.B. Galerkin, A.A. Drozdov, K.V. Soldatova. Experimental characteristic simulation for two-stage pipeline centrifugal compressor, International Conference on Compressors and their Systems 2019, IOP
Conf. Series: Materials Science and Engineering (London, 2019)

[13] A. Borovkov, I. Voinov, Y. Galerkin, A. Nikiforov, M. Nikitin, O. Solovyeva, K. Kabalyk, Issues of gas dynamic characteristics modeling: a study on a centrifugal compressor model stage, E3S Web of Conferences (2019)

[14] L. Marenina, Y. Galerkin, K. Soldatova, International Journal of Mechanical Engineering and Robotics Research 7, 6, 656-661 (2018)

[15] Y. Galerkin, O. Solovieva, Flow behavior and performances of centrifugal compressor stage vaneless diffusers, International Conference on Numerical Methods in Industrial Processes, International Journal of Mechanical, Aerospace, Industrial, Mechatronic and Manufacturing Engineering 9, 1 (France, Paris, 2015)

[16] O.A. Solovyova, A mathematical model for calculating the gas-dynamic characteristics and optimizing the vaneless diffusers of centrifugal compressor stages: PhD thesis (SPbPU. 2018) 